\title{
ON RAYLEIGH WAVES IN A THINLY LAYERED LAMINATED THERMOELASTIC MEDIUM WITH STRESS COUPLES UNDER INITIAL STRESSES*
}

\author{
Pijush Pal Roy \\ Blasting Department \\ Central Mining Research Station \\ Barwa Road, Dhanbad - 826001 \\ Bihar, India \\ and \\ Lokenath Debnath \\ Department of Mathematics \\ University of Central Florida \\ Orlando, Florida 32816, U.S.A
}

\begin{abstract}
A study is made of the propagation of Rayleigh waves in a thinly layered laminated thermoelastic medium under deviatoric, hydrostatic, and couple stresses. The frequency equation of the Rayleigh waves is obtained. The phase velocity of the Rayleigh waves depends on the initial stress, deviatoric stress, and the couple stress. The laminated medium is first replaced by an equivalent anisotropic thermoelastic continuum. The corresponding thermoelastic coefficients (after deformation) are derived in terms of initially isotropic thermoelastic coefficients (before deformation) of individual layers. Several particular cases are discussed for the determination of the displacement fields with or without the effect of the couple stress.
\end{abstract}

Key words: Rayleigh Waves, Incremental Stresses, Thermal Stresses, Couple Stresses.

1980 AMS Subject Classification Codes: 73D15, 73D20, 73K20

\section{INTRODUCTION}

Based upon the general ideas of incremental deformations, Biot [1-3] first developed an exact solution of the problems of buckling and dynamics of multilayered and laminated structures with stress couples under initial stresses. Subsequently, Tolstoy [4] modified Biot's formulation and obtained simple explicit solutions of the dynamical equations for a prestressed solid under horizontal compression in a gravitational field. $\mathrm{He}$ showed how the magnitudes of the effects of two factors, overburden pressure and deviatoric stress (or stress difference) influence seismic wave propagation velocities.

\footnotetext{
" Received April 21, 1988, Revised July 1, 1988.
} 
The laminated thermoelastic medium, which consists of a superposition of thin hard and soft adhering thermoelastic materials, is first approximated by an equivalent anisotropic medium. The corresponding composite incremental coefficients (after deformation) are obtained as functions of the coefficients before deformation and the initial stress components of individual layers. Pal Roy [5] investigated the problem of elastic wave propagation in a thinly layered laminated medium with stress couples under initial stresses. Further, Biot's analysis [2] also included the microelastic properties of the layers by the use of stress couples. This inclusion obviously makes a better approximation.

In his recent papers, Pal Roy [5-7] discussed some studies of elastic wave propagation in a thinly layered laminated medium with stress couples under initial stresses. This work deserves further extension in a thermoelastic medium.

Several authors including Lockett [8], Nowacki and Sokolowski [9] and Biot [3] have made some investigations of the thermoelastic wave propagation in different geometrical configurations. Among them, Biot's work includes the concept of generalized free energy along with a new definition of the dissipation function in terms of the time derivative of an entropy displacement. This work also represents a unified treatment of thermoelastic problems.

The main purpose of this work is to study the propagation of Rayleigh waves in a laminated thermoelastic medium under deviatoric, hydrostatic, and couple stresses. The frequency equation of these waves is derived. It is shown that the phase velocity of the Rayleigh waves depends on the initial stress, deviatoric stress, and the couple stresses. Several particular cases are discussed with or without the effect of the couple stresses.

\section{STRESS-STRAIN RELATIONS FOR A THERMOELASTIC LAMINATED MEDIUM}

We consider a thermoelastic laminated medium at an absolute temperature $T$. The medium is composed of thin adhering layers which are alternately hard and soft. The hard and soft materials are both compressible and they occupy fractions $\alpha_{1}$ and $\alpha_{2}$ of the total thickness. If the rigidity contrast of the layers is not too large, and the layer thickness remains sufficiently small with respect to the wavelength of the deformation field, then such a laminated medium may be approximated by a continuous medium of anisotropic properties. For simplicity and without loss of generality, we assume incremental deformations in the $x, y$ plane only. If we increase the absolute temperature by a small amount $\theta$, the incremental stresses for hard and soft materials can be written following Biot [1-3]. 
For the hard initially isotropic material, the incremental stresses $t_{\mathrm{ij}}$ related to strains $\mathrm{e}_{\mathrm{ij}}$ are given by

$$
t_{11}^{(1)}=a_{1} e_{x x}+b_{1} e_{y y}^{(1)}-\beta_{1} \cdot \theta
$$$$
t_{22}=b_{1} e_{x x}+c_{1} e_{y y}^{(1)}-\beta_{1} \cdot \theta
$$$$
t_{12}=2 L_{1} e_{x y}
$$

\begin{tabular}{|l|}
\hline initially isotropic \\
materials \\
\hline$\lambda . \mu . \mathrm{P}_{\mathrm{i}}$ \\
\hline \\
\hline
\end{tabular}

a. Thinly layered laminated thermoelastic medium.

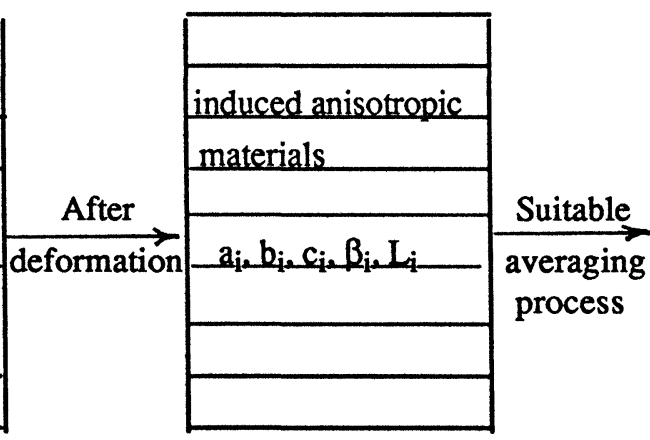

b. Layered structure after deformation.
$C_{11}, C_{12}, C_{22}, L, A$

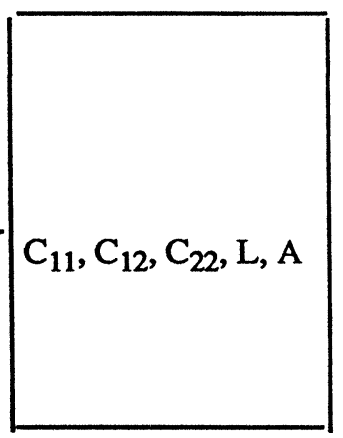

c. Equivalent anisotropic thermoelastic medium.

Fig. 1: Geometry of the problem.

For the soft material, the stress-strain relations are

$$
\begin{aligned}
& t_{11}^{(2)}=a_{2} e_{x x}+b_{2} e_{y y}^{(2)}-\beta_{2} \cdot \theta \\
& t_{22}=b_{2} e_{x x}+c_{2} e_{y y}^{(2)}-\beta_{2} \cdot \theta \\
& t_{12}=2 L_{2} e_{x y}
\end{aligned}
$$


where $a_{k}, b_{k}, c_{k}, L_{k}$ are the alternative elastic coefficients, $k=1$ (hard material), $k=2$ (soft material), and $\beta_{r}(r=1,2)$ are thermal coefficients. Since soft and hard materials are both compressible, the strain component $e_{y y}$ is not the same in each layer. Also we have

$$
\begin{array}{r}
e_{y y}=\alpha_{1} e_{y y}^{(1)}+\alpha_{2} e_{y y}^{(2)} \\
t_{11}=\alpha_{1} t_{11}^{(1)}+\alpha_{2} t_{11}^{(2)}
\end{array}
$$

where $\alpha_{1}+\alpha_{2}=1$.

When a uniform shear stress $t_{12}$ is applied, the total average shear strain is $e_{x y}=$ $(2 L)^{-1} t_{12}$, that is,

where $L=\left(\frac{\alpha_{1}}{L_{1}}+\frac{\alpha_{2}}{L_{2}}\right)^{-1}$ is the composite slide modulus. Since we are concerned with a uniform strain without shear, the normal strain component $e_{x x}$ and the normal stress are the same in both materials. Eliminating the four variables, $t_{11}^{(1)}, t_{11}^{(2)}, e_{y y}^{(1)}$ and $e_{y y}^{(2)}$, from equations (2.1)-(2.8), we obtain, for the composite material of anisotropic nature,

$$
\begin{aligned}
& t_{11}=C_{11} e_{x x}+C_{12} e_{y y}-A_{1} \cdot \theta \\
& t_{22}=C_{12} e_{x x}+C_{22} e_{y y}-A_{2} \cdot \theta
\end{aligned}
$$

where $C_{i j}$ and $A_{i}$ are the composite thermoelastic coefficients given by

$$
\begin{gathered}
C_{11}=\alpha_{1} a_{1}+\alpha_{2} a_{2}-\frac{\alpha_{1} \alpha_{2}\left(b_{1}-b_{2}\right)^{2}}{\alpha_{1} c_{2}+\alpha_{2} c_{1}}, C_{22}=\frac{c_{1} c_{2}}{\alpha_{2} c_{2}+\alpha_{2} c_{1}} \\
C_{12}=\frac{\alpha_{1} b_{1} c_{2}+\alpha_{2} b_{2} c_{1}}{\alpha_{1} c_{2}+\alpha_{2} c_{1}} \\
A_{1}=\frac{\left(\beta_{1} \alpha_{1}+\beta_{2} \alpha_{2}\right)\left(\alpha_{1} c_{2}+\alpha_{2} c_{1}\right)-\left(\beta_{1}-\beta_{2}\right)\left(b_{1}-b_{2}\right)}{\left(\alpha_{1} c_{2}+\alpha_{2} c_{1}\right)}
\end{gathered}
$$




$$
A_{2}=\frac{\alpha_{1} \beta_{1} c_{2}+\alpha_{2} \beta_{2} c_{1}}{\alpha_{1} c_{2}+\alpha_{2} c_{1}}
$$

If now we replace $A_{1}$ and $A_{2}$ by their average value (1/2)( $\left.A_{1}+A_{2}\right)$, the equations (2.9) (2.11) become

$$
\begin{aligned}
& t_{11}=C_{11} e_{x x}+C_{12} e_{y y}-A \cdot \theta \\
& t_{22}=C_{12} e_{x x}+C_{22} e_{y y}-A \cdot \theta \\
& t_{12}=2 L e_{x y}
\end{aligned}
$$

where

$$
A=\left[\left(\alpha_{1} \beta_{1}+\alpha_{2} \beta_{2}\right)\left(\alpha_{1} c_{2}+\alpha_{2} c_{1}\right)-\left(\beta_{1}-\beta_{2}\right)\left(b_{1}-b_{2}\right)\right.
$$

$$
\left.+\left(\alpha_{1} \beta_{1} c_{2}+\alpha_{2} \beta_{2} c_{1}\right)\right] /\left[2\left(\alpha_{1} c_{2}+\alpha_{2} c_{1}\right)\right]
$$

\section{THE INITIALLY ISOTROPIC HARD MATERIAL}

The state of initial stress introduces anisotropy (induced anisotropy) so that even for an initially isotropic solid defined by two Lame's constants $\lambda_{1}$ and $\mu_{1}$, the number of $B_{i j}^{1}$ will always be larger than 2 . The initial stress components $S_{i j}^{k}$ are

$$
S_{11}^{1}=-P_{1}-\rho_{1} g y
$$

$$
s_{22}^{1}=-\rho_{1} g y
$$

where $g$ is the acceleration due to gravity. The elastic coefficients $B_{i j}^{(\mathbf{k})}$ after deformation are obtained in [5] (see also Ref. [1], p. 111). 
(3.2ab)

$$
B_{11}^{1}=\left(2 \mu_{1}+\lambda_{1}\right)\left(1+\frac{S_{11}^{1}-S_{22}^{1}}{2 \mu_{1}}\right), B_{22}^{1}=\left(2 \mu_{1}+\lambda_{1}\right)\left(1+\frac{S_{22}^{1}-S_{11}^{1}}{2 \mu_{1}}\right)
$$

(3.3ab) $\mathrm{B}_{12}^{1}=\lambda_{1}-\mathrm{S}_{11}^{1}, \mathrm{Q}^{1}=\mu_{1}+\frac{1}{4}\left(\mathrm{~S}_{11}^{1}+\mathrm{S}_{22}^{1}\right)$

The alternative elastic coefficients $a_{\mathbf{k}}, b_{\mathbf{k}}, c_{\mathbf{k}}$ and $L_{\mathbf{k}}$ are the same in the references [5] and [1], and have the form

$$
\begin{aligned}
& a_{1}=B_{11}^{1}=\left(\lambda_{1}+2 \mu_{1}\right)\left(1-\frac{P_{1}}{2 \mu_{1}}\right), b_{1}=B_{12}^{1}-P_{1}-\rho_{1} g y=\lambda_{1} \\
& c_{1}=B_{22}^{1}=\left(2 \mu_{1}+\lambda_{1}\right)\left(1+\frac{P_{1}}{2 \mu_{1}}\right), L_{1}=\mu_{1}
\end{aligned}
$$

where $\mathbf{P}_{\mathbf{k}}$ denotes the compression.

\section{THE INITIALLY ISOTROPIC SOFT MATERIAL}

Following the above exactly, the alternative elastic coefficients $a_{2}, b_{2}, c_{2}$ and $L_{2}$ are obtained as (Ref. [5] and [1], p. 61)

$$
\begin{aligned}
& a_{2}=\left(\lambda_{2}+\mu_{2}\right)\left(1-\frac{P_{2}}{2 \mu_{2}}\right), b_{2}=\lambda_{2} \\
& c_{2}=\left(\lambda_{2}+\mu_{2}\right)\left(1+\frac{P_{2}}{2 \mu_{2}}\right), L_{2}=\mu_{2} .
\end{aligned}
$$

\section{THE COMPOSITE ANISOTROPIC MATERIAL}

From equations (2.12ab) - (2.15), (3.4ab) - (3.5ab), and (4.1ab) - (4.2ab), we derive the following:

$$
\begin{aligned}
& C_{11}=\alpha_{1}\left(\lambda_{1}+2 \mu_{1}\right)\left(1-\frac{P_{1}}{2 \mu_{1}}\right)+\alpha_{2}\left(\lambda_{2}+2 \mu_{2}\right)\left(1-\frac{P_{2}}{2 \mu_{2}}\right)-\frac{\alpha_{1} \alpha_{2}}{K}\left(\lambda_{1}-\lambda_{2}\right)^{2} \\
& C_{12}=\frac{1}{K}\left[\left(\lambda_{2}+2 \mu_{2}\right)\left(1+\frac{P_{2}}{2 \mu_{2}}\right) \alpha_{1} \lambda_{1}+\left(2 \mu_{1}+\lambda_{1}\right)\left(1+\frac{P_{1}}{2 \mu_{1}}\right) \alpha_{2} \lambda_{2}\right]
\end{aligned}
$$




$$
\begin{aligned}
& \mathrm{C}_{22}=\frac{1}{\mathrm{~K}}\left[\left(\lambda_{1}+2 \mu_{1}\right)\left(\lambda_{2}+2 \mu_{2}\right)\left(1+\frac{\mathrm{P}_{1}}{2 \mu_{1}}\right)\left(1+\frac{\mathrm{P}_{2}}{2 \mu_{2}}\right)\right] \\
& \mathrm{L}=1 /\left(\frac{\alpha_{1}}{\mu_{1}}+\frac{\alpha_{2}}{\mu_{2}}\right)
\end{aligned}
$$

$$
A=\frac{1}{2}\left(\alpha_{1} \beta_{1}+\alpha_{2} \beta_{2}\right)-\frac{1}{2 K}\left[\left(\beta_{1}-\beta_{2}\right)\left(\lambda_{1}-\lambda_{2}\right)\right.
$$

$$
\begin{aligned}
& \left.\quad+\alpha_{1} \beta_{1}\left(\lambda_{2}+2 \mu_{2}\right)\left(1+\frac{P_{2}}{2 \mu_{2}}\right)+\alpha_{2} \beta_{2}\left(\lambda_{1}+2 \mu_{1}\right)\left(1+\frac{P_{1}}{2 \mu_{1}}\right)\right] \\
& K=\alpha_{1}\left(\lambda_{2}+2 \mu_{2}\right)\left(1+\frac{P_{2}}{2 \mu_{2}}\right)+\alpha_{2}\left(\lambda_{1}+2 \mu_{1}\right)\left(1+\frac{P_{1}}{2 \mu_{1}}\right) .
\end{aligned}
$$

\section{COUPLE STRESS ANALOGY}

Following Biot [2], the couple stress of the composite material is

$$
M=b \frac{\partial^{2} v}{\partial x^{2}}
$$

where the couple stress coefficient, b, is

$$
b=\frac{1}{3} h^{\prime 2} \alpha_{1} \alpha_{2} \frac{\left(L_{1}-L_{2}\right)}{\left(\alpha_{1} L_{1}+\alpha_{2} L_{2}\right)}\left(M_{1} \alpha_{1}^{2}-M_{2} \alpha_{2}^{2}\right)
$$

and the total thickness $h^{\prime}$ is the sum of the thicknesses $h_{1}$ and $h_{2}$ of the layers so that

$$
h^{\prime}=h_{1}+h_{2}
$$

and

$$
M_{i}=\frac{1}{4 c_{i}}\left(a_{i} c_{i}-b_{i}^{2}\right), i=1,2 .
$$




\section{BASIC GOVERNING EQUATIONS}

The equations of equilibrium in terms of alternative stress components $t_{i j}$ are given by (Ref. [1], p. 61 and 264)

$$
\begin{aligned}
& \frac{\partial t_{11}}{\partial x}+\frac{\partial t_{12}}{\partial y}+\left(\frac{P}{4}+\frac{\rho g y}{2}\right) \frac{\partial^{2} v}{\partial x \partial y}+\left(\frac{P}{4}-\frac{\rho g y}{2}\right) \frac{\partial^{2} u}{\partial y^{2}}=\rho \frac{\partial^{2} u}{\partial t^{2}} \\
& \begin{aligned}
\frac{\partial t_{12}}{\partial x}+\frac{\partial t_{22}}{\partial y}+\frac{1}{2}\left(\frac{P}{2}+\rho g y\right) \frac{\partial^{2} u}{\partial x \partial y}-\frac{1}{2}\left(\frac{3}{2} P+\rho g y\right) \frac{\partial^{2} v}{\partial x^{2}}+g \rho\left(\frac{\partial u}{\partial x}+\frac{\partial v}{\partial y}\right) \\
\quad=\rho \frac{\partial^{2} v}{\partial t^{2}}+b \frac{\partial^{4} v}{\partial x^{4}}
\end{aligned}
\end{aligned}
$$

where $u$ and $v$ are the displacement fields, $P$ is the density, and $P$ is the average compression, while the effect of the couple stress of the composite medium has been incorporated in these equations.

Tolstoy [4] has shown, by taking a typical value of shear wave velocity $c_{s} \simeq 4 x$ $10^{3} \mathrm{~ms}^{-1}$, that the effect of gravity $(\mathrm{g})$ is approaching $1 \%$ of the inertial terms for periods $\mathrm{T} \geq 255$. If then one asumes $(\mathrm{P} / \mu) \geq 2 \times 10^{-2}$, the buoyancy term corrections will be appreciably smaller than those of prestress for periods of half a minute or less. As such, in dealing with typical seismic body wave frequencies, we shall therefore neglect the $g$ (buoyancy) terms in equation (7.2) in comparison with the prestress terms. (We, however, keep the hydrostatic pressure terms in equations (7.1) and (7.2).)

When we use relations (2.9) - (2.11) and drop the buoyancy term in (7.2), equations (7.1) - (7.2) become

$$
\begin{gathered}
C_{11} \frac{\partial^{2} u}{\partial x^{2}}+B \frac{\partial^{2} v}{\partial x \partial y}+D \frac{\partial^{2} u}{\partial y^{2}}=\rho \frac{\partial^{2} u}{\partial t^{2}}+A \frac{\partial \theta}{\partial x} \\
C_{22} \frac{\partial^{2} v}{\partial y^{2}}+B \frac{\partial^{2} u}{\partial x \partial y}+(D-P) \frac{\partial^{2} v}{\partial x^{2}}=\rho \frac{\partial^{2} v}{\partial t^{2}}+A \frac{\partial \theta}{\partial y}+b \frac{\partial^{4} v}{\partial x^{4}}
\end{gathered}
$$

where 
(7.5ab) $\quad \mathrm{B}=\mathrm{C}_{12}+\mathrm{L}+\frac{1}{4} \mathrm{P}+\frac{1}{2} \rho g y, \mathrm{D}=\mathrm{L}+\frac{1}{4} \mathrm{P}-\frac{1}{2} \rho g y$.

Equations (7.3) - (7.4) are the modified forms of the equations previously obtained by $\mathrm{Pal}$ Roy [5]. The equation of heat conduction is obtained from Biot [3] in the form

$$
\nabla^{2} \theta-\frac{\mathrm{a}}{\mathrm{K}} \frac{\partial \theta}{\partial \mathrm{t}}-\frac{\mathrm{TA}}{\mathrm{K}} \frac{\partial \mathrm{e}}{\partial \mathrm{t}}=0
$$

where $a$ is the specific heat of the unit volume in the absence of deformation, $k$ is the coefficient of heat conduction, $\mathrm{T}$ is the absolute temperature for the state of zero stressstrain, and

$$
e=e_{x x}+e_{y y} \equiv u_{x}+v_{y}
$$

We next express the displacement fields $u$ and $v$ in terms of the classical potentials $\phi$ and $\psi$ as

$$
u=\frac{\partial \phi}{\partial x}-\frac{\partial \psi}{\partial y}, \quad v=\frac{\partial \phi}{\partial y}+\frac{\partial \psi}{\partial x}
$$

Thus equation (7.6) can be written in terms of $\phi$ and $\theta$ as

$$
\nabla^{2} \theta-\frac{1}{\xi} \frac{\partial \theta}{\partial \mathrm{t}}-\eta \nabla^{2}\left(\frac{\partial \phi}{\partial \mathrm{t}}\right)=0
$$

where $\xi=\frac{\kappa}{a}$ and $\eta=\frac{T A}{\kappa}$.

In view of (7.7ab), equations (7.3) - (7.4) assume the form

$$
\begin{aligned}
& \frac{\partial^{2} \phi}{\partial \mathrm{x}^{2}}+\frac{(\mathrm{B}+\mathrm{D})}{\mathrm{C}_{11}} \frac{\partial^{2} \phi}{\partial \mathrm{y}^{2}}=\frac{\rho}{\mathrm{C}_{11}} \frac{\partial^{2} \phi}{\partial \mathrm{t}^{2}}+\frac{\mathrm{A} \cdot \theta}{\mathrm{C}_{11}} \\
& \frac{\partial^{2} \phi}{\partial \mathrm{x}^{2}}+\frac{\mathrm{C}_{22}}{(\mathrm{~B}+\mathrm{D}-\mathrm{P})} \frac{\partial^{2} \phi}{\partial \mathrm{y}^{2}}=\frac{\rho}{(\mathrm{B}+\mathrm{D}-\mathrm{P})} \frac{\partial^{2} \phi}{\partial \mathrm{t}^{2}}+\frac{\mathrm{b}}{(\mathrm{B}+\mathrm{D}-\mathrm{P})} \frac{\partial^{4} \phi}{\partial \mathrm{x}^{4}}+\frac{\mathrm{A} \cdot \theta}{(\mathrm{B}+\mathrm{D}-\mathrm{P})} \\
& \frac{\partial^{2} \psi}{\partial \mathrm{x}^{2}}+\frac{\left(\mathrm{C}_{22}-\mathrm{B}\right)}{(\mathrm{D}-\mathrm{P})} \frac{\partial^{2} \psi}{\partial \mathrm{y}^{2}}=\frac{\rho}{(\mathrm{D}-\mathrm{P})} \frac{\partial^{2} \psi}{\partial \mathrm{x}^{2}}+\frac{\mathrm{b}}{(\mathrm{D}-\mathrm{P})} \frac{\partial^{4} \psi}{\partial \mathrm{x}^{4}}
\end{aligned}
$$




$$
\frac{\partial^{2} \psi}{\partial x^{2}}+\frac{D}{\left(C_{11}-B\right)} \frac{\partial^{2} \psi}{\partial y^{2}}=\frac{\rho}{\left(C_{11}-B\right)} \frac{\partial^{2} \psi}{\partial t^{2}} .
$$

Plane wave solutions. We consider compressional and distortional waves along the $\mathrm{x}$ direction only. Eliminating $\theta$ from equations (7.8) and (7.9), we obtain (7.13) and retain (7.11) so that

$$
\begin{aligned}
& \left(\nabla^{2}-\frac{1}{\xi} \frac{\partial^{2}}{\partial \mathrm{t}^{2}}\right)\left(\frac{\partial^{2}}{\partial \mathrm{x}^{2}}+\frac{\mathrm{B}+\mathrm{D}}{\mathrm{C}_{11}} \frac{\partial^{2}}{\partial \mathrm{y}^{2}}-\frac{\rho}{\mathrm{C}_{11}} \frac{\partial^{2}}{\partial \mathrm{t}^{2}}\right) \phi-\eta \mathrm{m} \nabla^{2}\left(\frac{\partial \phi}{\partial \mathrm{t}}\right)=0 \\
& \frac{\partial^{2} \Psi}{\partial \mathrm{x}^{2}}+\frac{\mathrm{C}_{22}-\mathrm{B}}{\mathrm{D}-\mathrm{p}} \frac{\partial^{2} \Psi}{\partial \mathrm{y}^{2}}=\frac{\rho}{\mathrm{D}-\mathrm{p}} \frac{\partial^{2} \Psi}{\partial \mathrm{t}^{2}}+\frac{\mathrm{b}}{\mathrm{D}-\mathrm{p}} \frac{\partial^{4} \Psi}{\partial \mathrm{x}^{4}}
\end{aligned}
$$

For a plane harmonic wave propagating in the $\mathrm{x}$ direction, we seek solutions in the form

$$
\phi(x, y, t)=\Phi(y) \exp [i \omega(t-x / c)]
$$

$$
\Psi(x, y, t)=\Psi(y) \exp [i \omega(t-x / c)]
$$

where $\mathrm{c}$ is the phase velocity of the Rayleigh waves in the thermoelastic medium under initial stresses.

Putting equations (7.15ab) into equations (7.13) - (7.14), we obtain

$$
\begin{aligned}
& {\left[\left(D^{2}-\alpha^{2}-q\right)\left(D^{2}-\alpha_{1}^{2}+\sigma^{2}\right)-q \varepsilon\left(D^{2}-\alpha^{2}\right)\right] \Phi(y)=0} \\
& \left(D^{2}-\alpha_{2}^{2}+\tau^{2}\right) \Psi(y)=0
\end{aligned}
$$

where

$$
\begin{aligned}
& D \equiv \frac{d}{d y}, \quad q=\frac{i \omega}{\xi}, \quad \alpha^{2}=\omega^{2} / c^{2}, \\
& \alpha_{1}^{2} \equiv \alpha^{2} C_{11} /(B+D), \quad \sigma^{2}=\rho \omega^{2} /(B+D), \quad \varepsilon=m \eta \xi C_{11} /(B+D) \\
& \alpha_{2}^{2}=\alpha^{2}(D-P) /\left(C_{22}-B\right), \tau^{2}=\frac{\rho \omega^{2}}{C_{22}-B}\left(1-\frac{b \alpha^{2}}{\rho c^{2}}\right) .
\end{aligned}
$$

The solutions of equations (7.16) - (7.17), satisfying the boundary conditions in the form of the corresponding stresses decaying exponentially with depth, have the form: 


$$
\Phi(y)=A_{1} e^{-\lambda_{1} y}+B_{1} e^{-\lambda_{2} y}
$$

(7.18ab)

$$
\Psi(y)=C_{1} e^{-\gamma y}
$$

where $\lambda_{1}$ and $\lambda_{2}$ are the roots of the biquadratic equation

$$
\lambda^{4}-\lambda^{2}\left(\alpha^{2}+\alpha_{1}^{2}+q_{1}-\sigma^{2}\right)+\left(\alpha^{2}-q\right)\left(\alpha_{1}^{2}-\sigma^{2}\right)+q \varepsilon \alpha^{2}=0
$$

and

$$
\begin{aligned}
& q_{1}=q(1+\varepsilon) \\
& \gamma=\left(\alpha_{2}^{2}-\tau^{2}\right)^{1 / 2}=\left(\alpha^{2} e^{2}-\tau^{2}\right)^{1 / 2} \\
& e^{2}=(D-p) /\left(C_{22}-B\right) .
\end{aligned}
$$

From equations (7.15ab) and (7.18ab), we obtain

$$
\phi=\left(A_{1} e^{-\lambda_{1} y}+B_{1} e^{-\lambda_{2} y}\right) \exp [i \omega(t-x / c)]
$$

(7.23ab)

$$
\psi=C_{1} e^{-\gamma y} \exp [i \omega(t-x / c)]
$$

and $\theta$ can be found from (7.9) in the form

$$
\theta=\frac{1}{m} e^{i \omega(t-x / c)}\left[\eta_{1} A_{1} e^{-\lambda_{1} y}+\eta_{2} B_{1} e^{-\lambda 2_{2}^{y}}\right]
$$

where

$$
\eta_{1}=d^{2}\left(\lambda_{1}^{2}-\alpha_{1}^{2}+\sigma^{2}\right), \quad \eta_{2}=d^{2}\left(\lambda_{2}^{2}-\alpha_{1}^{2}+\sigma^{2}\right)
$$

and

$$
d^{2}=(B+D) / C_{11}
$$




\section{THE FREQUENCY EQUATION FOR THE RAYLEIGH WAVES}

We set the boundary conditions on the plane $y=0$ as (Biot [1] and Nowacki [10])

$$
e_{x y}=0, \quad t_{22}=0, \quad \frac{\partial \theta}{\partial y}+h \theta=0
$$

where $h$ is the ratio of the coefficients of heat transfer to the thermal conductivity.

We next use (2.9) - (2.11), (7.7ab), and (7.23ab) - (7.24) to rewrite the boundary conditions $(8.1 \mathrm{abc})$ in the form

$$
\begin{aligned}
& 2 \alpha \lambda_{1} A_{1}+2 \alpha \lambda_{2} B_{1}+i\left(\gamma^{2}+\alpha^{2}\right) C_{1}=0 \\
& \left(\lambda_{1}^{2} C_{22}-C_{12} \alpha^{2}-\frac{A \eta_{1}}{m}\right) A_{1}+\left(\lambda^{2} C_{22}-\alpha^{2} C_{12}-\frac{A \eta_{2}}{m}\right) B_{1}+\gamma i k\left(C_{22}-C_{12}\right) C_{1}=0 \\
& A_{1} \eta_{1}\left(\lambda_{1}-h\right)+B_{1} \eta_{2}\left(\lambda_{2}-h\right)=0
\end{aligned}
$$

Eliminating $A_{1}, B_{1}$ and $C_{1}$ from these results, we get

$$
\left|\begin{array}{ccc}
2 \alpha \lambda_{1} & 2 \alpha \lambda_{2} & \gamma^{2}+\alpha^{2} \\
\lambda_{1}^{2} C_{22}-C_{12} \alpha^{2}-\frac{A \eta_{1}}{m} & \lambda_{2}^{2} C_{22}-\alpha^{2} C_{12}-\frac{A \eta_{2}}{m} & \gamma \cdot \alpha\left(C_{22}-C_{12}\right) \\
\eta_{1}\left(\lambda_{1}-h\right) & \eta_{2}\left(\lambda_{2}-h\right) & 0
\end{array}\right|=0
$$

Expanding this determinant, we obtain the frequency equation for the Rayleigh waves

$$
\begin{aligned}
& \eta_{1}\left(\lambda_{1}-h\right)\left[2 \gamma \lambda_{2} \alpha^{2}\left(C_{22}-C_{12}\right)-\left(\gamma^{2}+\alpha^{2}\right)\left(\lambda_{2}^{2} C_{22}-\alpha^{2} C_{12}-\frac{A \eta_{2}}{m}\right)\right] \\
& +\eta_{2}\left(\lambda_{2}-h\right)\left[\left(\gamma^{2}+\alpha^{2}\right)\left(\lambda_{1}^{2} C_{12}-C_{12} \alpha^{2}-\frac{A \eta_{1}}{m}\right)\right. \\
& \left.\quad-2 \alpha^{2} \gamma \lambda_{1}\left(C_{22}-C_{12}\right)\right]=0 .
\end{aligned}
$$

It is clear from equation (8.6) that the phase velocity of the Rayleigh waves depends on the initial stress, deviatoric stress, and couple stress. 


\section{SOME PARTICULAR CASES OF INTEREST}

We assume a convection condition (8.1c) for the temperature on the boundary. In the particular case $h=0$, we deal with thermal insulation; while for $h \rightarrow \infty$, the temperature vanishes on the boundary. In the particular case of the uncoupled problem $(\varepsilon=0)$, we have

$$
\begin{aligned}
& \lambda_{1}=\left(\alpha^{2}+q\right)^{\frac{1}{2}}, \quad \lambda_{2}=\left(\frac{\alpha^{2}}{d^{2}}-\sigma^{2}\right)^{\frac{1}{2}} \\
& \eta_{1}=\left(d^{2}-1\right) \alpha^{2}+d^{2}\left(\sigma^{2}+q\right), \quad \eta_{2}=0 .
\end{aligned}
$$

Equation (8.6) now becomes

$$
2 \sqrt{\frac{1}{d^{2}}-\frac{\sigma^{2}}{\alpha^{2}}} \sqrt{e^{2}-\frac{\tau^{2}}{\alpha^{2}}}\left(C_{22}-C_{12}\right)-\left(1+e^{2}-\frac{\tau^{2}}{\alpha^{2}}\right)\left[\left(\frac{1}{d^{2}}-\frac{\sigma^{2}}{\alpha^{2}}\right) C_{22}-C_{12}\right]=0,
$$

when there is no couple stress $(b=0)$ and equation (9.3) reduces to the form

$$
2\left\{\left(\frac{1}{d^{2}}-\frac{X^{2}}{\mathscr{L}^{2}}\right)\left(\mathrm{e}^{2}-\mathrm{X}^{2}\right)\right\}^{\frac{1}{2}}\left(1-\frac{\mathrm{C}_{12}}{\mathrm{C}_{22}}\right)-\left(1+\mathrm{e}^{2}-\mathrm{X}^{2}\right)\left(\frac{1}{\mathrm{~d}^{2}}-\frac{\mathrm{X}^{2}}{\mathscr{L}^{2}}-\frac{\mathrm{C}_{12}}{\mathrm{C}_{22}}\right)=0,
$$

where

$$
X=\frac{c}{c_{1}}, c_{1}^{2}=\frac{C_{22}-B}{\rho}=\frac{C_{22}-C_{12}-L-\frac{P}{4}}{\rho}, \mathscr{L}^{2}=\frac{\tau^{2}}{\sigma^{2}}
$$

We consider a thinly layered laminated thermoelastic medium of $50 \mathrm{~cm}$. in thickness. The hard and soft materials occupy respectively $30 \mathrm{~cm}$. and $20 \mathrm{~cm}$. of the total. The following dimensions are taken into account: 


$$
\begin{aligned}
& \lambda_{1}=\mu_{1}=6 \times 10^{11} \text { dynes } / \mathrm{cm}^{2}, \quad \beta_{1}=4 \times 10^{11} \text { dynes } / \mathrm{cm}^{2} \\
& \lambda_{2}=\mu_{2}=3 \times 10^{11} \text { dynes } / \mathrm{cm}^{2}, \quad \beta_{2}=2.5 \times 10^{11} \text { dynes } / \mathrm{cm}^{2} \\
& \alpha_{1}=\frac{3}{5}, \quad \alpha_{2}=\frac{2}{5}, \quad \frac{P_{1}}{2 \mu_{1}}=0.48, \quad \frac{P_{2}}{2 \mu_{2}}=0.32 .
\end{aligned}
$$

Then from formulas (5.4), (5.6), and (6.4) and the above results we obtain Tables 1 and 2.

\section{Table 1}

\begin{tabular}{|c|c|c|c|}
\hline \multicolumn{4}{|c|}{$10^{11}$ dynes $/ \mathrm{cm}^{2}$} \\
\hline $\mathrm{L}$ & $\mathrm{K}$ & $\mathrm{M}_{1}$ & $\mathrm{M}_{2}$ \\
\hline 4.2857143 & 17.784 & 2.0021622 & 1.3406061 \\
\hline
\end{tabular}

Table 2 Non-Dimensional Values of Constants

\begin{tabular}{|c|c|c|c|c|c|c|}
\hline $\mathrm{C}_{11} / \mathrm{L}$ & $\mathrm{C}_{12} / \mathrm{L}$ & $\mathrm{C}_{22} / \mathrm{L}$ & $\mathrm{A} / \mathrm{L}$ & $\mathrm{b} / \mathrm{L}$ & $\mathrm{P} / 2 \mathrm{~L}$ & $\mathrm{~m}$ \\
\hline 1.8532599 & 0.9805668 & 4.1523886 & 0.00533738 & 14.766541 & 0.9856 & 0.00288 \\
\hline
\end{tabular}

We have, from equation (9.4),

$$
\text { (9.6) } \mathrm{X}^{8}-8.1808195 \mathrm{X}^{6}+19.231638 \mathrm{X}^{4}-12.643484 \mathrm{X}^{2}+9.3293485=0 \text {. }
$$

The corresponding equation when the effect of couple stress is taken into account becomes

$$
\text { (9.7) } \mathrm{X}^{8}-8.1869549 \mathrm{X}^{6}+19.274874 \mathrm{X}^{4}-12.725369 \mathrm{X}^{2}+9.346593=0
$$

The equation (9.6) has a real root 1.8698823 (when $b=0$ ). The corresponding root of equation (9.7) is 1.8697807 (when $b \neq 0$ ). This shows the measure of the structure's couple stress effect on the velocity of Rayleigh wave propagation. Assuming the effect of hydrostatic pressure is small, i.e. at a depth of $y \mathrm{~cm} . M=\frac{\rho g y}{L}$ is small, we can express the functions $\phi$ and $\psi$.

(a) In the absence of couple stress $(b=0)$, we find

$$
\phi=B_{1} \exp [-i \alpha(0.2530411-1.9891009 M) y+i \omega(t-x / c)]
$$




$$
\psi=\mathrm{B}_{1}(-0.2143526+0.7331583 \mathrm{M}) \times
$$

$$
\exp [-\mathrm{i} \alpha(1.8333055+0.1224613 \mathrm{M}) \mathrm{y}+\mathrm{i} \omega(\mathrm{t}-\mathrm{x} / \mathrm{c})] \text {. }
$$

(b) In the presence of couple stress $(b \neq 0)$, we obtain

$$
\begin{aligned}
\phi= & B_{1} \exp [-i \alpha y(0.2530411-1.9891009 M)+i \omega(t-x / c)] \\
\Psi= & B_{1}\left(-0.2009407+0.6738628 M-0.2005658 x^{2}+0.5684386 M \alpha^{2}\right) \times \\
& x \exp \left[-i \alpha y\left(1.9556071-1.9520222 \alpha^{2}-1.013815 M \alpha^{2}\right)+i \omega(t-x / c)\right] .
\end{aligned}
$$

The displacement components can be easily be obtained from equations (7.7ab), (9.7) (9.8) and (9.9) - (9.11).

\section{ACKNOWLEDGMENT}

The authors express their sincere thanks to the Department of Applied Mathematics, University of Calcutta, for providing facilities for numerical computations. This work was partially supported by the University of Central Florida. We are grateful to the referee for suggesting some improvements.

\section{REFERENCES}

[1] Biot, M. A., Mechanics of Incremental Deformations, John Wiley and Sons, New York, 1965.

[2] Biot, M. A., Buckling and Dynamics of Multilayered and Laminated Plates Under Initial Stress, Int. J Solids Structs., 10 (1974), 414-451.

[3] Biot, M. A., Thermoelasticity and Irreversible Thermodynamics, J. App. Phy., 27 (1956), 240-253.

[4] Tolstoy, I., On Elastic Waves in Prestressed Solids, Jr. Geoph. Res., 87 (1982), 6823-6827.

[5] Pal Roy, P., On Elastic Waves in a Thinly Layered Laminated Medium with Stress Couples Under Initial Stresses, Proc. Int. Sym. on Waves and Stability in Continuous Media, Bari, Oct. 7-12, Italy. 
[6] Pal Roy, P., A Simplified Approach on the Propagation of Edge Waves in a Thinly Layered Laminated Medium with Stress Couples Under Biaxial Initial Stresses, Acta Geoph. Pol., 33 (1985).

[7] Pal Roy, P., Wave Propagation in a Thinly Two-layered Laminated Medium with Stress Couples Under Initial Stresses, Acta Mech., 54 (1984), 1-21.

[8] Lockett, F. J., Effect of Thermal Properties of a Solid on the Velocity of Rayleigh Waves, J. Mech. Phys. Solids, 1958.

[9] Nowacki, W. and Sokolowski, M., Propagation of Thermoelastic Waves in Plates, Arch. Mech. Stos., 11 (1959).

[10] Nowacki, W., Thermoelasticity, Addison-Wesley Publishing Company, London, 1962. 


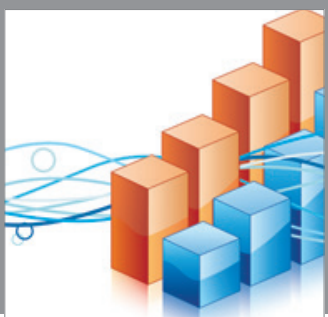

Advances in

Operations Research

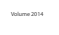

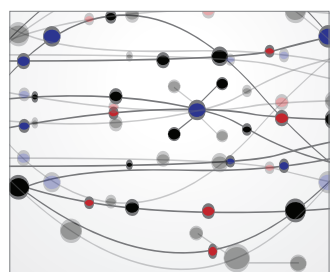

\section{The Scientific} World Journal
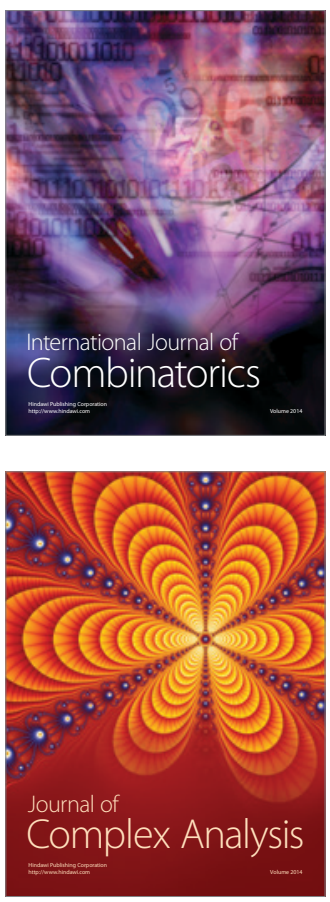

International Journal of

Mathematics and

Mathematical

Sciences
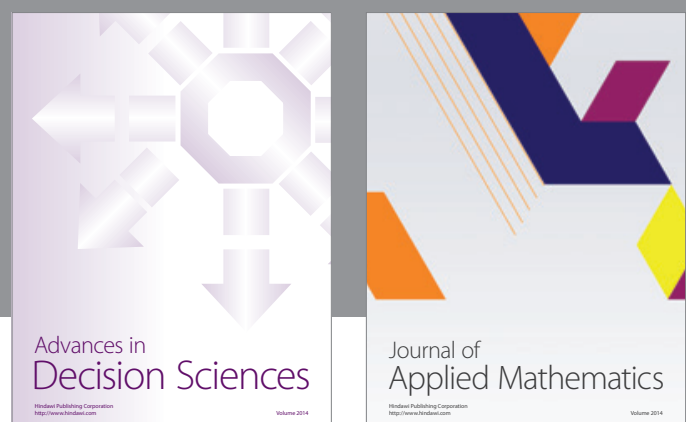

Journal of

Applied Mathematics
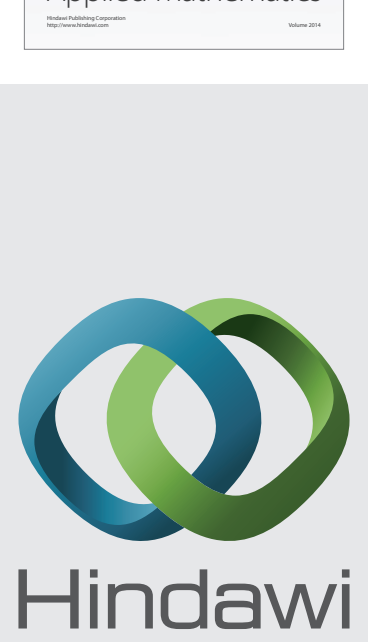

Submit your manuscripts at http://www.hindawi.com
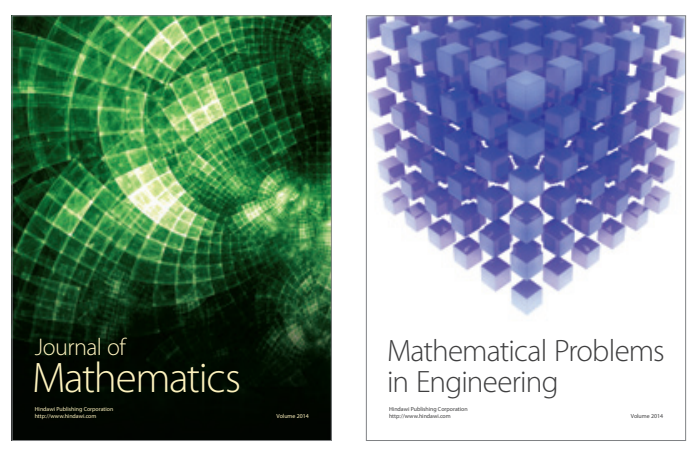

Mathematical Problems in Engineering
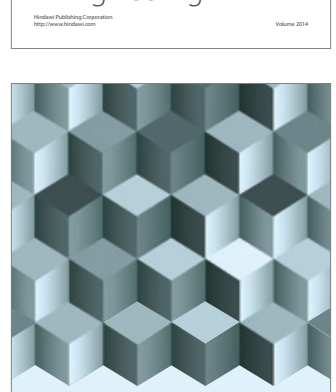

Journal of

Function Spaces
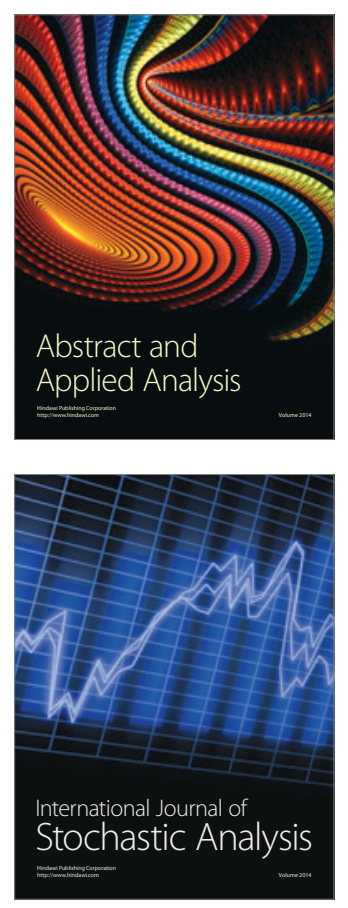

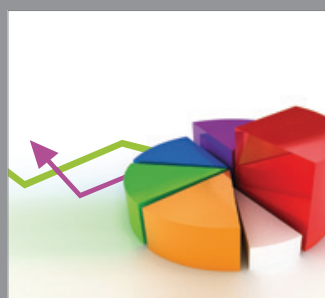

ournal of

Probability and Statistics

Promensencen
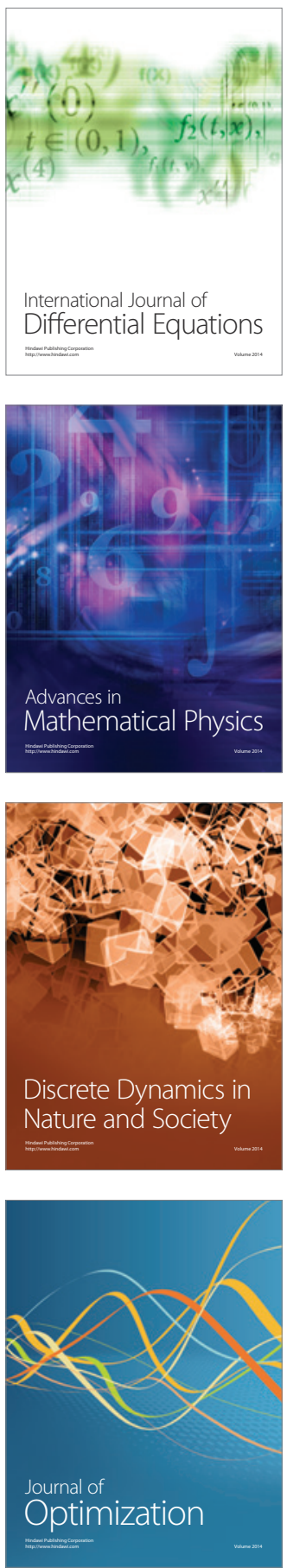